\title{
LIE SOLVABLE RINGS
}

\author{
R. K. SHARMA AND J. B. SRIVASTAVA
}

\begin{abstract}
Let $\mathscr{L}(R)$ denote the associated Lie ring of an associative ring $R$ with identity $1 \neq 0$ under the Lie multiplication $[x, y]=x y-y x$ with $x, y \in R$. Further, suppose that the Lie ring $\mathscr{L}(R)$ is solvable of length $n$. It has been proved that if 3 is invertible in $R$, then the ideal $J$ of $R$ generated by all elements [[[ $\left.\left.\left.x_{1}, x_{2}\right],\left[x_{3}, x_{4}\right]\right], x_{5}\right], x_{1}, x_{2}, x_{3}, x_{4}, x_{5} \in R$, is nilpotent of index at most $\frac{2}{9}\left(19 \cdot 10^{n-3}-1\right)$ for $n \geqslant 3$. Also, if 2 and 3 are both invertible in $R$, then the ideal $I$ of $R$ generated by all elements $[x, y], x, y \in R$, is a nil ideal of $R$. Some applications to Lie solvable group rings are also given.
\end{abstract}

Let $R$ be any associative ring with identity $1 \neq 0$. We can induce the Lie structure on $R$ by defining the Lie product $[x, y]=x y-y x$ for $x, y \in R$. The Lie ring thus obtained is called the associated Lie ring of $R$ and is denoted by $\mathscr{L}(R)$. Jennings [1] proved that if $\mathscr{L}(R)$ is nilpotent then the associative ideal of $R$ generated by all elements [ $[x, y], z], x, y, z \in R$, is a nilpotent ideal of $R$ and the ideal generated by all $[x, y], x, y \in R$, is nil. In this paper we study the case when $\mathscr{L}(R)$ is solvable.

1. Lie identities and Lie ideals. Let $x_{1}, x_{2}, \ldots, x_{n} \in R$; then the left normed commutators are defined by $\left[x_{1}, x_{2}\right]=x_{1} x_{2}-x_{2} x_{1}$ and, inductively,

$$
\left[x_{1}, x_{2}, \ldots, x_{n}\right]=\left[\left[x_{1}, x_{2}, \ldots, x_{n-1}\right], x_{n}\right] \text {. }
$$

We shall repeatedly use the following well-known identities, which are easy to prove.

LEMMA 1.1 For $x, y, z \in R$, the following identities are true:

(i) $[x, y]=-[y, x]$.

(ii) $[x, y, z]+[y, z, x]+[z, x, y]=0$ (Jacobi identity).

(iii) $[x y, z]=x[y, z]+[x, z] y$.

(iv) $[x, y z]=y[x, z]+[x, y] z$.

For any two subsets $A$ and $B$ of $R$, by $[A, B]$ we shall denote the additive subgroup of $R$ generated by all elements $[a, b]$ with $a \in A$ and $b \in B$. A Lie ideal of $R$ means an ideal of the Lie ring $\mathscr{L}(R)$. Thus, $U$ is a Lie ideal if $U$ is an additive subgroup of $R$ and $[u, r] \in U$ for $u \in U$ and $r \in R$. It is easy to see that $[U, V]$ is a Lie ideal if $U$ and $V$ are Lie ideals.

Received by the editors April 27, 1984.

1980 Mathematics Subject Classification. Primary 16A68.

Key words and phrases. Lie solvable ring, associated Lie ring. 
Let $U$ be any Lie ideal of $R$. In view of the identity $u r=[u, r]+r u, u \in U$, $r \in R$, the right and left ideals of $R$, generated by $U$, are identical. In particular, $R U=U R$ is the two-sided ideal generated by $U$. Also, $U^{m}$ consists of finite sums of $m$-fold products $u_{1} u_{2} \cdots u_{m}$ with $u_{1}, u_{2}, \ldots, u_{m} \in U$. Therefore, $(U R)^{n}=U^{n} R$ for any Lie ideal $U$ and for any positive integer $n$.

The derived chain of any Lie ideal $U$ is given by

$$
U=\delta^{(0)}(U) \supseteq \delta^{(1)}(U) \supseteq \delta^{(2)}(U) \supseteq \cdots \supseteq \delta^{(n)}(U) \supseteq \cdots,
$$

where $\delta^{(n+1)}(U)=\left[\delta^{(n)}(U), \delta^{(n)}(U)\right], n \geqslant 0$. We say that $\mathscr{L}(R)$ is solvable of length $n$ if $\delta^{(n)}(\mathscr{L}(R))=(0), n$ least.

Further, the lower central chain of $U$ is defined by

$$
U=\gamma_{1}(U) \supseteq \gamma_{2}(U) \supseteq \gamma_{3}(U) \supseteq \cdots \supseteq \gamma_{n}(U) \supseteq \cdots,
$$

where $\gamma_{n+1}(U)=\left[\gamma_{n}(U), U\right], n \geqslant 1$. The Lie ring $\mathscr{L}(R)$ is nilpotent of class $n$ if $\gamma_{n+1}(\mathscr{L}(R))=(0), n$ least.

We proceed with a sequence of lemmas needed for our further work. These lemmas are also of independent interest.

LEMMA 1.2. Let $U$ be a Lie ideal of $R$; then

(i) $\left[U^{m}, \mathscr{L}(R)\right] \subseteq[U, \mathscr{L}(R)] \subseteq U$, and

(ii) $\left[\delta^{(1)}(U) R, \delta^{(1)}(U) R\right] \subseteq U$.

Proof. (i) follows by induction on $m$ and the identity $\left[u_{1} u_{2}, r\right]=\left[u_{1}, u_{2} r\right]+$ $\left[u_{2}, r u_{1}\right]$.

To prove (ii), for $u_{1}, u_{2}, u_{3}, u_{4} \in U$ and $r, s \in R$, we have the identity

$$
\begin{aligned}
{\left[\left[u_{1}, u_{2}\right] r,\left[u_{3}, u_{4}\right] s\right]=} & {\left[\left[u_{1}, u_{2} r\right],\left[u_{3}, u_{4} s\right]\right]-\left[u_{2}\left[u_{1}, r\right], u_{4}\left[u_{3}, s\right]\right] } \\
& -\left[u_{2}\left[u_{1}, r\right],\left[u_{3}, u_{4}\right] s\right]+\left[u_{4}\left[u_{3}, s\right],\left[u_{1}, u_{2}\right] r\right] .
\end{aligned}
$$

This can easily be obtained by expanding the first term on the right and using Lemma 1.1.

Now the right side belongs to $U$ by (i). The lemma follows easily.

LeMMA 1.3. Let $U$ be a Lie ideal of $R$; then

(i) $\left(\delta^{(1)}(U)\right)^{2} \cdot \delta^{(1)}(\mathscr{L}(R)) \subseteq U+\left[\delta^{(1)}(U), \mathscr{L}(R)\right] R$, and

(ii) $\left(\delta^{(1)}(U)\right)^{4} \cdot \delta^{(2)}(\mathscr{L}(R)) \subseteq \delta^{(1)}(U)+\left[\delta^{(1)}(U), \mathscr{L}(R)\right] R$.

Proof. Let $v_{1}, v_{2}$ be two-fold commutators in the elements of $U$ and let $r_{1}, r_{2} \in R$. Expanding the first term on the right and using Lemma 1.1, we get the identity

$$
v_{1} v_{2}\left[r_{1}, r_{2}\right]=\left[v_{1} r_{1}, v_{2} r_{2}\right]-\left[v_{1}, v_{2} r_{2}\right] r_{1}-v_{1}\left[r_{1}, v_{2}\right] r_{2} \text {. }
$$

By Lemma 1.2(ii), $\left[v_{1} r_{1}, v_{2} r_{2}\right] \in U$, and the other two terms clearly belong to $\left[\delta^{(1)}(U), \mathscr{L}(R)\right] R$. This proves (i).

Now let $w_{1}, w_{2}, w_{3}, w_{4}$ be two-fold commutators in the elements of $U$, and let $s_{1}, s_{2}$ be two-fold commutators in the elements of $\mathscr{L}(R)=R$. Then the following identity can be obtained by using Lemma 1.1 and expanding the first term on the right:

$$
\begin{aligned}
w_{1} w_{2} w_{3} w_{4}\left[s_{1}, s_{2}\right]= & {\left[w_{1} w_{2} s_{1}, w_{3} w_{4} s_{2}\right]-w_{1} w_{2} w_{3}\left[s_{1}, w_{4}\right] s_{2} } \\
& -w_{1} w_{2}\left[s_{1}, w_{3}\right] w_{4} s_{2}-w_{1}\left[w_{2}, w_{3} w_{4} s_{2}\right] s_{1}-\left[w_{1}, w_{3} w_{4} s_{2}\right] w_{2} s_{1} .
\end{aligned}
$$


The first term on the right, by (i), easily belongs to $\delta^{(1)}(U)+\left[\delta^{(1)}(U), \mathscr{L}(R)\right] R$, and all other terms on the right are clearly in $\left[\delta^{(1)}(U), \mathscr{L}(R)\right] R$ because it is a two-sided ideal. Thus we get (ii).

This leads to our next lemma. Let $J$ denote the ideal of $R$ generated by all elements $\left[\left[x_{1}, x_{2}\right],\left[x_{3}, x_{4}\right], x_{5}\right]$ with $x_{1}, \ldots, x_{5} \in R$. Clearly,

$$
J=\left[\delta^{(1)}(\mathscr{L}(R)), \delta^{(1)}(\mathscr{L}(R)), \mathscr{L}(R)\right] R=\left[\delta^{(2)}(\mathscr{L}(R)), \mathscr{L}(R)\right] R .
$$

LEMMA 1.4. For any Lie ideal $U$ of $R$, we have

$$
\left(\delta^{(1)}(U)\right)^{4} \cdot J \subseteq\left[\delta^{(1)}(U), \mathscr{L}(R)\right] R .
$$

Proof. The left side is a finite sum of elements of the type $a[b, c] r$, with $a \in\left(\delta^{(1)}(U)\right)^{4}, b \in \delta^{(2)}(\mathscr{L}(R)), c \in \mathscr{L}(R)$, and $r \in R$. But

$$
a[b, c]=[a b, c]-[a, c] b .
$$

By Lemma 1.3(ii), $a b \in \delta^{(1)}(U)+\left[\delta^{(1)}(U), \mathscr{L}(R)\right] R$. Hence,

$$
[a b, c] \in\left[\delta^{(1)}(U), \mathscr{L}(R)\right] R \text {. }
$$

Also, by Lemma 1.2(i),

$$
[a, c] b \in\left[\left(\delta^{(1)}(U)\right)^{4}, \mathscr{L}(R)\right] R \subseteq\left[\delta^{(1)}(U), \mathscr{L}(R)\right] R .
$$

Thus, $a[b, c] r \in\left[\delta^{(1)}(U), \mathscr{L}(R)\right] R$ and the lemma is proved.

LEMmA 1.5. For any Lie ideal $U$ of $R$, we have

$$
\delta^{(1)}(U)\left[\delta^{(1)}(U), \mathscr{L}(R)\right] \subseteq \gamma_{3}(U) R .
$$

Proof. Let $u_{1}, u_{2}, u_{3}, u_{4} \in U$ and $r \in R$. Then the following identity gives the result:

$$
\begin{aligned}
{\left[u_{1}, u_{2}\right]\left[u_{3}, u_{4}, r\right]=} & {\left[\left[u_{3}, u_{4}\right],\left[u_{1}, u_{2} r\right]\right]-u_{2}\left[\left[u_{3}, u_{4}\right],\left[u_{1}, r\right]\right] } \\
& -\left[\left[u_{3}, u_{4}\right],\left[u_{1}, u_{2}\right]\right] r-\left[u_{3}, u_{4}, u_{2}\right]\left[u_{1}, r\right] .
\end{aligned}
$$

COROLlaRY 1.6. Let $U$ be a Lie ideal of $R$; then

(i) $\left[\delta^{(1)}(U), \mathscr{L}(R)\right]^{2} \subseteq \gamma_{3}(U) R$, and

(ii) $J^{2} \subseteq \gamma_{3}\left(\delta^{(1)}(\mathscr{L}(R))\right) R$.

Proof. (i) follows by Lemma 1.5 since $\left[\delta^{(1)}(U), \mathscr{L}(R)\right] \subseteq \delta^{(1)}(U)$.

(ii) follows from (i) if we put $U=\delta^{(1)}(\mathscr{L}(R))$ and observe that

$$
J=\left[\delta^{(2)}(\mathscr{L}(R)), \mathscr{L}(R)\right] R=R\left[\delta^{(2)}(\mathscr{L}(R)), \mathscr{L}(R)\right] .
$$

The next lemma is crucial to our further work. Its proof also requires some computations.

LEMMA 1.7. Let $U$ be a Lie ideal of a ring $R$ in which 3 is invertible. Then $\left(\gamma_{3}(U)\right)^{2} \subseteq \delta^{(2)}(U) R$.

Proof. It is enough to show that $\left[u_{1}, u_{2}, u_{3}\right]\left[u_{4}, u_{5}, u_{6}\right] \in \delta^{(2)}(U) R$ for all $u_{1}, \ldots, u_{6} \in U$. To do this, we proceed as follows. Let

$$
a=\left[u_{1}, u_{2}, u_{5}\right]\left[u_{6}, u_{4}, u_{3}\right]+\left[u_{1}, u_{2}, u_{6}\right]\left[u_{5}, u_{4}, u_{3}\right] \text {. }
$$


Observe that the second term can be obtained from the first by interchanging $u_{5}$ (the last entry of the first bracket) and $u_{6}$ (the first entry of the second bracket). Expanding $\left[\left[u_{4}, u_{6} u_{5}, u_{3}\right],\left[u_{1}, u_{2}\right]\right]$ properly, we can easily get

$$
\begin{aligned}
a= & {\left[\left[u_{4}, u_{6} u_{5}, u_{3}\right],\left[u_{1}, u_{2}\right]\right]+\left[\left[u_{1}, u_{2}, u_{5}\right],\left[u_{6}, u_{4}, u_{3}\right]\right] } \\
& -u_{6}\left[\left[u_{4}, u_{5}, u_{3}\right],\left[u_{1}, u_{2}\right]\right]-\left[\left[u_{4}, u_{6}, u_{3}\right],\left[u_{1}, u_{2}\right]\right] u_{5} \\
& -\left[u_{6}, u_{3}\right]\left[\left[u_{4}, u_{5}\right],\left[u_{1}, u_{2}\right]\right]-\left[\left[u_{6}, u_{3}\right],\left[u_{1}, u_{2}\right]\right]\left[u_{4}, u_{5}\right] \\
& -\left[u_{4}, u_{6}\right]\left[\left[u_{5}, u_{3}\right],\left[u_{1}, u_{2}\right]\right]-\left[\left[u_{4}, u_{6}\right],\left[u_{1}, u_{2}\right]\right]\left[u_{5}, u_{3}\right] .
\end{aligned}
$$

Certainly, $a \in \delta^{(2)}(U) R$.

In an exactly similar manner,

$$
b=\left[u_{1}, u_{2}, u_{4}\right]\left[u_{6}, u_{5}, u_{3}\right]+\left[u_{1}, u_{2}, u_{6}\right]\left[u_{4}, u_{5}, u_{3}\right] \in \delta^{(2)}(U) R .
$$

We now turn to the case when the last entry of the first bracket and the last entry of the second bracket are interchanged.

Expanding $\left[\left[u_{6}, u_{4}\right],\left[\left[u_{1}, u_{2}\right], u_{3} u_{5}\right]\right]$ and rearranging terms, we get

$$
\begin{aligned}
c= & {\left[u_{1}, u_{2}, u_{3}\right]\left[u_{6}, u_{4}, u_{5}\right]+\left[u_{1}, u_{2}, u_{5}\right]\left[u_{6}, u_{4}, u_{3}\right] } \\
= & {\left[\left[u_{6}, u_{4}\right],\left[\left[u_{1}, u_{2}\right], u_{3} u_{5}\right]\right]+\left[\left[u_{1}, u_{2}, u_{5}\right],\left[u_{6}, u_{4}, u_{3}\right]\right] } \\
& -u_{3}\left[\left[u_{6}, u_{4}\right],\left[u_{1}, u_{2}, u_{5}\right]\right]-\left[\left[u_{6}, u_{4}\right],\left[u_{1}, u_{2}, u_{3}\right]\right] u_{5} .
\end{aligned}
$$

This shows that $c \in \delta^{(2)}(U) R$.

Arguments, as in the case of $c$, will also give

$$
d=\left[u_{1}, u_{2}, u_{3}\right]\left[u_{6}, u_{5}, u_{4}\right]+\left[u_{1}, u_{2}, u_{4}\right]\left[u_{6}, u_{5}, u_{3}\right] \in \delta^{(2)}(U) R
$$

and

$$
e=\left[u_{1}, u_{2}, u_{3}\right]\left[u_{4}, u_{5}, u_{6}\right]+\left[u_{1}, u_{2}, u_{6}\right]\left[u_{4}, u_{5}, u_{3}\right] \in \delta^{(2)}(U) R .
$$

Finally, by using Lemma 1.1(ii) and rearranging terms, we get

$$
3\left[u_{1}, u_{2}, u_{3}\right]\left[u_{4}, u_{5}, u_{6}\right]=a-b-c+d+2 e \in \delta^{(2)}(U) R .
$$

Since 3 is invertible in $R$,

$$
\left[u_{1}, u_{2}, u_{3}\right]\left[u_{4}, u_{5}, u_{6}\right] \in \delta^{(2)}(U) R .
$$

This completes the proof.

COROllary 1.8. If 3 is invertible in $R$, then $J^{4} \subseteq \delta^{(3)}(\mathscr{L}(R)) R$.

Proof. $J^{2} \subseteq \gamma_{3}\left(\delta^{(1)}(\mathscr{L}(R))\right) R$ by Corollary 1.6. Therefore,

$$
\begin{aligned}
J^{4} & \subseteq\left(\gamma_{3}\left(\delta^{(1)}(\mathscr{L}(R))\right) R\right)^{2}=\left(\gamma_{3}\left(\delta^{(1)}(\mathscr{L}(R))\right)\right)^{2} R \\
& \subseteq \delta^{(2)}\left(\delta^{(1)}(\mathscr{L}(R))\right) R \quad(\text { by Lemma } 1.7) \\
& =\delta^{(3)}(\mathscr{L}(R)) R .
\end{aligned}
$$

The next lemma does not assume that 3 is invertible in $R$ and has a much simpler proof than Lemma 1.7.

LeMma 1.9. Let $U$ be a Lie ideal of $R$ such that $U$ is also a subring of $R$; then $\left(\gamma_{3}(U)\right)^{2} \subseteq \delta^{(2)}(U) R$. 
Proof. Expanding the first term on the right, we have

$$
\begin{aligned}
{\left[u_{1}, u_{2}, u_{3}\right]\left[u_{4}, u_{5}, u_{6}\right]=} & {\left[\left[u_{4}, u_{5}\right],\left[\left[u_{1}, u_{2}\right] u_{6}, u_{3}\right]\right] } \\
& +\left[\left[u_{1}, u_{2}, u_{3}\right],\left[u_{4}, u_{5}\right]\right] u_{6}+\left[u_{1}, u_{2}\right]\left[\left[u_{6}, u_{3}\right],\left[u_{4}, u_{5}\right]\right] \\
& +\left[\left[u_{1}, u_{2}\right],\left[u_{4}, u_{5}\right]\right]\left[u_{6}, u_{3}\right]
\end{aligned}
$$

for all $u_{1}, \ldots, u_{6} \in U$. Clearly the right side belongs to $\delta^{(2)}(U) R$, as desired.

COROllaRY 1.10. For any ring $R,\left(\gamma_{3}(\mathscr{L}(R))\right)^{2} \subseteq \delta^{(2)}(\mathscr{L}(R)) R$.

2. Main results. In this section we prove our main theorems.

THEOREM 2.1. Let $R$ be a ring in which 3 is invertible, and let its associated Lie ring $\mathscr{L}(R)$ be solvable of length $n \geqslant 3$. Then the ideal $J$ of $R$, generated by all elements $\left[\left[x_{1}, x_{2}\right],\left[x_{3}, x_{4}\right], x_{5}\right]$ with $x_{1}, \ldots, x_{5}$ in $R$, is nilpotent of index at most $\frac{2}{9}\left(19 \cdot 10^{n-3}-1\right)$.

Proof. If $\mathscr{L}(R)$ is solvable of length $n=3$, then $\delta^{(3)}(\mathscr{L}(R))=(0)$. By Corollary $1.8, J^{4}=(0)$. So the theorem is true for $n=3$.

We assume that $n \geqslant 4$. Now for any Lie ideal $U$ of $R$, using Lemmas 1.4 and 1.5, we get

$$
\delta^{(1)}(U)\left(\delta^{(1)}(U)\right)^{4} J \subseteq \gamma_{3}(U) R
$$

Thus, by Lemma 1.7,

$$
\left\{\left(\delta^{(1)}(U)\right)^{5} J\right\}^{2} \subseteq\left(\gamma_{3}(U) R\right)^{2}=\left(\gamma_{3}(U)\right)^{2} R \subseteq \delta^{(2)}(U) R .
$$

Putting $U=\delta^{(m-2)}(\mathscr{L}(R))$, we get

$$
\left\{\left(\delta^{(m-1)}(\mathscr{L}(R))\right)^{5} J\right\}^{2} \subseteq \delta^{(m)}(\mathscr{L}(R)) R \text { for all } m \geqslant 4 .
$$

Thus, for $m=4$, we have

$$
\left\{\left(\delta^{(3)}(\mathscr{L}(R))\right)^{5} J\right\}^{2} \subseteq \delta^{(4)}(\mathscr{L}(R)) R
$$

and, using Corollary 1.8 ,

$$
\left\{\left(J^{4}\right)^{5} J\right\}^{2}=J^{(1+2 \cdot 10) 2} \subseteq \delta^{(4)}(\mathscr{L}(R)) R .
$$

We claim that, by induction on $m$,

$$
J^{2\left\{1+10+10^{2}+\cdots+10^{m-4}+2 \cdot 10^{m-3}\right\}} \subseteq \delta^{(m)}(\mathscr{L}(R)) R \text { for all } m \geqslant 4 .
$$

Assume this is true for $m$ and use $\left\{\left(\delta^{(m)}(\mathscr{L}(R))\right)^{5} J\right\}^{2} \subseteq \delta^{(m+1)}(\mathscr{L}(R)) R$ to prove it for $m+1$. Thus,

$$
J^{N} \subseteq \delta^{(n)}(\mathscr{L}(R)) R=(0)
$$

where

$$
N=2\left\{1+10+10^{2}+\cdots+10^{n-4}+2 \cdot 10^{n-3}\right\}=\frac{2}{9}\left[19 \cdot 10^{n-3}-1\right],
$$

as desired. 
Next, we prove that the ideal $I$ of $R$, generated by all elements $[x, y], x, y \in R$, is a nil ideal if 2 and 3 are both invertible in $R$. First, we need the following

LEMMA 2.2. Let $R$ be a ring in which 2 is invertible. Then

(i) $\left[\left[x_{1}, x_{2}\right],\left[x_{3}, x_{4}\right]\right]^{3} \in J$ for all $x_{1}, \ldots, x_{4} \in R$,

(ii) $[x, y, z]^{10} \in J$ for all $x, y, z \in R$, and

(iii) $[x, y]^{21} \in J$ for all $x, y \in R$.

Proof. Expanding the first term on the right, we get

$$
\begin{aligned}
2\left[\left[x_{1}, x_{2}\right],\left[x_{3}, x_{4}\right]\right]^{2}= & {\left[\left[x_{1}, x_{2}\right]^{2},\left[x_{3}, x_{4}\right],\left[x_{3}, x_{4}\right]\right] } \\
& -\left[x_{1}, x_{2}\right]\left[\left[x_{1}, x_{2}\right],\left[x_{3}, x_{4}\right],\left[x_{3}, x_{4}\right]\right] \\
& -\left[\left[x_{1}, x_{2}\right],\left[x_{3}, x_{4}\right],\left[x_{3}, x_{4}\right]\right]\left[x_{1}, x_{2}\right] .
\end{aligned}
$$

Thus,

$$
2\left[\left[x_{1}, x_{2}\right],\left[x_{3}, x_{4}\right]\right]^{2} \equiv\left[\left[x_{1}, x_{2}\right]^{2},\left[x_{3}, x_{4}\right],\left[x_{3}, x_{4}\right]\right](\bmod J) .
$$

Also,

$$
\begin{aligned}
& 2\left[\left[x_{1}, x_{2}\right]^{2},\left[x_{3}, x_{4}\right],\left[x_{3}, x_{4}\right]\right]\left[\left[x_{1}, x_{2}\right],\left[x_{3}, x_{4}\right]\right] \\
&=\left[\left[x_{3}, x_{4}\right]^{2},\left[x_{1}, x_{2}\right]^{2},\left[x_{3}, x_{4}\right],\left[x_{1}, x_{2}\right]\right] \\
&+\left[\left[\left[x_{1}, x_{2}\right]^{2},\left[x_{3}, x_{4}\right],\left[x_{3}, x_{4}\right]\right],\left[\left[x_{1}, x_{2}\right],\left[x_{3}, x_{4}\right]\right]\right] \\
&+\left[\left[x_{1}, x_{2}\right]^{2},\left[x_{3}, x_{4}\right],\left[x_{3}, x_{4}\right],\left[x_{1}, x_{2}\right]\right]\left[x_{3}, x_{4}\right] \\
&+\left[x_{3}, x_{4}\right]\left[\left[x_{1}, x_{2}\right]^{2},\left[x_{3}, x_{4}\right],\left[x_{3}, x_{4}\right],\left[x_{1}, x_{2}\right]\right] \\
& \equiv 0(\bmod J) .
\end{aligned}
$$

Combining, we get $4\left[\left[x_{1}, x_{2}\right],\left[x_{3}, x_{4}\right]\right]^{3} \in J$, and, since 2 is invertible, we get (i).

To prove (ii), observe the identity

$$
[x, y, z]^{2}=[[x, y],[[x, y] z, z]]+[[x, y, z],[x, y]] z
$$

and use (i), keeping in view that

$$
r\left[\left[x_{1}, x_{2}\right],\left[x_{3}, x_{4}\right]\right]=\left[\left[x_{1}, x_{2}\right],\left[x_{3}, x_{4}\right]\right] r-\left[\left[x_{1}, x_{2}\right],\left[x_{3}, x_{4}\right], r\right] .
$$

Similarly, to prove (iii) it is enough to use (i) and see that

$$
[x, y]^{3}=[[x y, y],[y x, x]]+[[y, x],[x y, y]] x+[[y x, x],[x, y]] y .
$$

Note that powers given in Lemma 2.2 are not the best possible; the purpose is served by proving that some power in each case belongs to $J$.

THEOREM 2.3. Let $R$ be a ring in which both 2 and 3 are invertible, and let $I_{0}$ be the ideal of $R$ generated by all elements $[x, y, z], x, y, z \in R$. If the associated Lie ring $\mathscr{L}(R)$ is solvable, then $I_{0}$ is a nil ideal.

Proof. Clearly, $I_{0}=\gamma_{3}(\mathscr{L}(R)) R=R \gamma_{3}(\mathscr{L}(R))$. By Corollary $1.10, I_{0}^{2}=$ $\left(\gamma_{3}(\mathscr{L}(R))\right)^{2} R \subseteq \delta^{(2)}(\mathscr{L}(R)) R$. Now suppose $\mathscr{L}(R)$ is solvable of length $n$, so $\delta^{(n)}(\mathscr{L}(R))=(0)$. If $n=1, I_{0}=(0)$. If $n=2, I_{0}^{2}=(0)$. So assume $n \geqslant 3$. 
Let $\alpha \in I_{0}$; then $\alpha^{2} \in I_{0}^{2} \subseteq \delta^{(2)}(\mathscr{L}(R)) R$ and, hence,

$$
\alpha^{2}=\sum_{i=1}^{m}\left[\left[x_{i}, y_{i}\right],\left[u_{i}, v_{i}\right]\right] r_{i}=\sum_{i=1}^{m} \alpha_{i} r_{i},
$$

where $\alpha_{i}=\left[\left[x_{i}, y_{i}\right],\left[u_{i}, v_{i}\right]\right]$ for $i=1,2, \ldots, m$. By Lemma 2.2(i) each $\alpha_{i}^{3} \in J$. Also, $\left[\alpha_{i}, r\right] \in J$ for every $r \in R$. Further, $\alpha_{i} \beta \alpha_{i}=\alpha_{i}^{2} \beta-\alpha_{i}\left[\alpha_{i}, \beta\right]$ for any $\beta \in R$. Thus,

$$
\alpha_{i} \beta \alpha_{i} \equiv \alpha_{i}^{2} \beta \quad(\bmod J)
$$

and, similarly,

$$
\alpha_{i} \beta \alpha_{i} \theta \alpha_{i} \equiv \alpha_{i}^{3} \beta \theta(\bmod J) \equiv 0(\bmod J)
$$

as $\alpha_{i}^{3} \in J$.

The above arguments, applied to $\left(\alpha^{2}\right)^{k}=\left(\sum_{1}^{m} \alpha_{i} r_{i}\right)^{k}$ for $k \geqslant 2 m+1$, immediately give that $\left(\alpha^{2}\right)^{k} \in J$ for $k \geqslant 2 m+1$. But, by Theorem 2.1, $J$ is nilpotent, hence $\left(\left(\alpha^{2}\right)^{k}\right)^{N}=0$ for suitable $N$. This proves that $\alpha$ is nilpotent for every $\alpha \in I_{0}$. That is, $I_{0}$ is a nil ideal.

In fact, we are able to obtain a much stronger result.

THEOREM 2.4. Let $R$ be a ring in which both 2 and 3 are invertible, and let $I$ be the ideal of $R$ generated by all elements $[x, y], x, y \in R$. If the associated Lie ring $\mathscr{L}(R)$ is solvable, then I is a nil ideal.

Proof. $I=\gamma_{2}(\mathscr{L}(R)) R=\delta^{(1)}(\mathscr{L}(R)) R$. Let $\mathscr{L}(R)$ be solvable of length $n$; then $\delta^{(n)}(\mathscr{L}(R))=(0)$. Therefore, if $n=1, I=(0)$.

Let $\alpha \in I$; then

$$
\alpha=\sum_{i=1}^{m}\left[x_{i}, y_{i}\right] r_{i}=\sum_{i=1}^{m} \alpha_{i} r_{i},
$$

where $\alpha_{i}=\left[x_{i}, y_{i}\right]$. By Lemma 2.2(iii), $\alpha_{i}^{21}=\left[x_{i}, y_{i}\right]^{21}$ always belongs to $J$.

Now,

$$
[x, y] r[x, y]=[x, y]^{2} r-[x, y][x, y, r] \equiv[x, y]^{2} r \quad\left(\bmod I_{0}\right) .
$$

If we take $\alpha^{k}=\left(\sum_{i=1}^{m} \alpha_{i} r_{i}\right)^{k}$ for $k \geqslant 20 m+1$, then $\alpha^{k}$ will be a finite sum of $k$-fold products of elements from $\left\{\alpha_{1} r_{1}, \alpha_{2} r_{2}, \ldots, \alpha_{m} r_{m}\right\}$, and in each $k$-fold product some $\alpha_{i}$ will be repeated at least 21 times. Collecting repeatedly these factors, by the above process, the $k$-fold products will be congruent to $\alpha_{i}^{21} r\left(\bmod I_{0}\right)$. Since $\alpha_{i}^{21} \in J$, this implies that $\alpha^{k}=\lambda+\mu$ with $\lambda \in J$ and $\mu \in I_{0}$. But, by Theorem 2.3, $I_{0}$ is a nil ideal, so $\mu^{l}=0$ for some $l$. This gives $\left(\alpha^{k}\right)^{l}=\alpha^{k l} \in J$. Now use the nilpotency of $J$ to get $\left(\alpha^{k l}\right)^{N}=0$. Thus, $\alpha$ is nilpotent and $I$ is a nil ideal.

3. Applications to group rings. Lie solvable group rings were studied by Passi, Passman and Sehgal in [2]. Let $K[G]$ denote the group ring of the group $G$ over the field $K$ with Char $K=p \geqslant 0, p \neq 2$. If $p>0$ we say that a group $G$ is $p$-Abelian if the commutator subgroup $G^{\prime}$ is a finite $p$-group. For convenience, 0 -Abelian will mean Abelian. It was proved in [2] that if Char $K=p \neq 2$, then the associated Lie algebra $\mathscr{L}(K[G])$ of $K[G]$ is solvable if and only if $G$ is $p$-Abelian. Using Theorem 2.4 we get an alternative proof in characteristic 0 as follows. 
Suppose Char $K=0$ and $\mathscr{L}(K[G])$ is solvable. Then, by Theorem $2.4, I=$ $\gamma_{2}(\mathscr{L}(K[G])) \cdot K[G]$ is a nil ideal of $K[G]$. So, by [3, Theorem 2.3 .4 , p. 47], $I=(0)$. This gives $\gamma_{2}(\mathscr{L}(K[G]))=(0)$, i.e., $K[G]$ is commutative. Hence, $G$ is Abelian. Thus, $\mathscr{L}(K[G])$ is solvable if and only if $G$ is Abelian.

Also in Char $K=p>0, p \neq 2,3$, we have an advantage. Suppose $\mathscr{L}(K[G])$ is solvable. Then $I=[K[G], K[G]] K[G]=\omega\left(K\left[G^{\prime}\right]\right) \cdot K[G]$ is a nil ideal of $K[G]$ by Theorem 2.4. Hence, $\omega\left(K\left[G^{\prime}\right]\right) \cdot K[G]$ is contained in the Jacobson radical $J(K[G])$ of $K[G]$. By [3, Lemma 10.1.13, p. 415], $G^{\prime}$ is a p-group. Also, $\mathscr{L}(K[G])$ is solvable implies $K[G]$ satisfies a polynomial identity. By [3, Theorem 5.2.14, p. 189], $|G: \Delta(G)|<\infty$ and $\Delta^{\prime}(G)$ is finite, where $\Delta(G)$ is the FC-subgroup of $G$. Now $G^{\prime} / G^{\prime} \cap \Delta(G)$ is a finite $p$-group, $G^{\prime} \cap \Delta(G) / \Delta^{\prime}(G)$ is Abelian, and $\Delta^{\prime}(G)$ is a finite $p$-group implies $G^{\prime}$, hence $G$, is solvable. Thus, $G^{\prime}$ is a locally finite $p$-group, since it is a solvable $p$-group. Also, as in [2], if it is proved that $G$ is an FC-group, then $G$ is $p$-Abelian by the above argument. Perhaps our results lead to a different motivation.

\section{REFERENCES}

1. S. A. Jennings, On rings whose associated Lie rings are nilpotent, Bull. Amer. Math. Soc. 53 (1947), 593-597.

2. I. B. S. Passi, D. S. Passman and S. K. Sehgal, Lie solvable group rings, Canad. J. Math. 25 (1973), $748-757$.

3. D. S. Passman, The algebraic structure of group rings, Wiley, New York, 1977.

Department of Mathematics, Indian Institute of Technology, New Delhi 110016, India 